\title{
Environmentally Friendly Flame-Retardant and Its Application in Rigid Polyurethane Foam
}

\author{
Yongjun Chen, Zhixin Jia, Yuanfang Luo, Demin Jia, and Bin Li \\ Department of Polymer Materials and Engineering, South China University of Technology, Guangzhou 510640, China \\ Correspondence should be addressed to Yongjun Chen; psyjchen@126.com and Zhixin Jia; zxjia@scut.edu.cn
}

Received 16 June 2014; Revised 18 September 2014; Accepted 26 September 2014; Published 28 October 2014

Academic Editor: Jose Ramon Leiza

Copyright (C) 2014 Yongjun Chen et al. This is an open access article distributed under the Creative Commons Attribution License, which permits unrestricted use, distribution, and reproduction in any medium, provided the original work is properly cited.

\begin{abstract}
A novel Flame-Retardant N-(P,P'-diphenyl) phosphorus-based-(3-triethoxysilicon) propylamine (DPTP) was synthesized in this study. The impact of DPTP on the mechanical properties, thermal stability, and flame retardancy of rigid polyurethane foam (RPUF) was studied. The addition of DPTP to RPUF can significantly reduce the undesirable thermal effects and smoke density during combustion, as well as increasing the limiting oxygen index. Compared with pure RPUF, the peak heat release rate of RPUF containing $10 \mathrm{phr}$ of DPTP decreased by $39.4 \%$, while its peak smoke production rate decreased by $49.9 \%$. However, it was also found that the addition of DPTP reduced the compressive strength of RPUF.
\end{abstract}

\section{Introduction}

Rigid polyurethane foam (RPUF) has the lowest thermal conductivity of all foams currently available; moreover, it possesses further advantages such as high compressive strength, good adhesive strength, and ease of processing $[1,2]$. Therefore, it will be significantly advantageous if RPUF can replace traditional inorganic thermal insulation materials for use as energy-efficient building materials [3]. However, compared to inorganic thermal insulation materials, RPUF has some limitations such as low density, large surface area, high flammability, and production of toxic smoke. In addition, the production of a large amount of smoke in widely used halogen flame retardants containing RPUF has limited the application of RPUF in energy-efficient buildings, especially for high-rise buildings. Intumescent flame-retardant polymer systems composed of an acid source, a carbon source, and a gas source with ammonium polyphosphate (APP) [4-6] as the main component and composite ones using expandable graphite (EG) [7-9] as the main component have advantages such as good flame retardancy, low smoke production, and low toxicity. The latter, in particular, has been widely used for manufacturing low smoke and flame-retardant RPUF. In recent years, owing to the increasing demand for RPUF in the field of energy-efficient building materials, flame-retardant
RPUF has again attracted considerable attention from domestic and international industry and academia [10-12]. This has led to a number of studies on the application of EG mixed with composites such as phosphorus-containing flame retardants, silica whiskers, or methyl methacrylate-acrylic acid copolymer in RPUF. In this study, we designed and synthesized a new type of halogen-free P-, N-, Si-containing flame retardant, namely, $\mathrm{N}-\left(\mathrm{P}, \mathrm{P}^{\prime}\right.$-diphenyl) phosphorus-based-(3triethoxysilicon) propylamine (DPTP), and applied it to RPUF. We investigated the flame retardancy of DPTP in RPUF, as well as the flame-retardant mechanism of DPTP.

\section{Experimental}

2.1. Main Raw Materials. $\gamma$-Aminopropyl triethoxysilane (KH-550): WD Silicone Co., Ltd. (Hubei); chlorodiphenyl phosphine (DPC): Qingdao Fusilin Chemical Technology Co., Ltd.; toluene: Tianjin Fuyu Fine Chemicals Co., Ltd.; triethylamine: Tianjin Damao Chemical Reagent Co., Ltd.; polyether polyols: industrial grade, brand HF-4110, hydroxyl value $=400-460 \mathrm{mg} \mathrm{KOH} / \mathrm{g}$, viscosity $=500-6500 \mathrm{mPa} \cdot \mathrm{s}$ $\left(25^{\circ} \mathrm{C}\right)$ : Hengfeng Polyurethane Industry Co., Ltd. (Zhejiang); polyether polyols: industrial grade, $\mathrm{HF}-4110 \mathrm{H}$ hydroxyl value $=400-460 \mathrm{mg} \mathrm{KOH} / \mathrm{g}$, viscosity $=1500-2000 \mathrm{mPa} \cdot \mathrm{s}\left(25^{\circ} \mathrm{C}\right)$ : 
TABLE 1: Formulations of RPUF.

\begin{tabular}{lccccccc}
\hline \multirow{2}{*}{ Sample } & & \multicolumn{2}{c}{ Polyol } & \multirow{2}{*}{ PAPI } & DPTP & Blowing agent (n-pentane) & \multicolumn{2}{c}{$\begin{array}{c}\text { Catalyst, N,N-dimethylcyclohexylamine } \\
\text { (DMCHA) }\end{array}$} \\
\hline 1\# & Neat PU & 30 & 70 & 138 & 0 & 30 & 0.5 \\
$2 \#$ & DPTP 2 phr & 30 & 70 & 138 & 2 & 30 & 0.5 \\
$3 \#$ & DPTP 5 phr & 30 & 70 & 138 & 5 & 0.5 & 0.5 \\
$4 \#$ & DPTP 2 phr & 30 & 70 & 138 & 8 & 0.5 & 0.5 \\
5\# & DPTP 2 phr & 30 & 70 & 138 & 10 & 0.5 & 0.5 \\
\hline
\end{tabular}

TABLE 2: Data obtained from cone calorimeter testing of RPUF.

\begin{tabular}{lcccc}
\hline Sample & TTI & PHRR $\left(\mathrm{kW} / \mathrm{m}^{2}\right)$ & PSPR $\left(\mathrm{m}^{2} / \mathrm{s}\right)$ & AMLR $(\mathrm{g} / \mathrm{s})$ \\
\hline $1 \#$ & 7 & 361.07 & 0.4498 & 0.0672 \\
$2 \#$ & 5 & 274.75 & 0.1975 & 0.0519 \\
3\# & 5 & 244.23 & 0.1813 & 0.0454 \\
$4 \#$ & 4 & 244.58 & 0.2137 & 0.0487 \\
5\# & 4 & 221.59 & 0.2235 & 0.0446 \\
\hline
\end{tabular}

TTI: time to ignition; PHRR: peak release rate; PSPR: peak smoke release rate; AMLR: average mass loss rate.

Hengfeng Polyurethane Industry Co., Ltd. (Zhejiang); polyarylpolymethylene-isocyanate (PAPI): industrial grade, functionality $=2.7$, NCO content $=30.75 \%$ : Huntsman Chemical Trading Co., Ltd.

2.2. Preparation of DPTP. First, $22.1 \mathrm{~g}(0.1 \mathrm{~mol})$ of $\gamma$ aminopropyl triethoxysilane (KH-550) and $50 \mathrm{~mL}$ of dry toluene were weighed out and placed in a $250 \mathrm{~mL}$ fourneck round-bottom flask that was purged with nitrogen. Thereafter, $10.1 \mathrm{~g}$ of triethylamine $(0.1 \mathrm{~mol})$ was added with continuous stirring. Then, $22 \mathrm{~g}(0.1 \mathrm{~mol})$ of DPC was dissolved in $25 \mathrm{~mL}$ of dry toluene, and the resulting solution was added dropwise to the four-neck round-bottom flask by using a constant-pressure dropping funnel for $1.5 \mathrm{~h}$. When the addition was complete, the temperature was raised to $85^{\circ} \mathrm{C}$. The reaction was continued under nitrogen for $10 \mathrm{~h}$. After the reaction was completed, followed by cooling, filtration, washing with cyclohexane, and removing the solvent by reduced pressure distillation, a novel flame-retardant synergist, DPTP, was obtained as a viscous light green product with $91 \mathrm{wt} \%$ yield (Figure 2).

2.3. Preparation of Flame-Retardant RPUFs. The foams were prepared from polyols and polymeric isocyanate. Blowing agent, surfactant, and catalyst were added and mixed with the polyols and, subsequently, the isocyanate was mixed and the mixture was poured into a $10.5 \mathrm{~cm} \times 10.5 \mathrm{~cm} \times 12.0 \mathrm{~cm}$ steel box. The $\mathrm{NCO} / \mathrm{OH}$ ratio used was $1.2: 1.0$. The obtained $\mathrm{PU}$ foams were put in an oven for complete cure for $24 \mathrm{~h}$ at $80^{\circ} \mathrm{C}$ in order to complete the polymerization reaction, before carrying out characterization. The compositions of polyurethane foams are shown in Table 1.
2.4. Measurements and Characterization. Fourier transform infrared spectroscopy (FTIR) analysis was performed using a Magna 760 Fourier transform infrared spectrometer (Nicolet, USA) within the wave number range of $400-4000 \mathrm{~cm}^{-1}$. Thermal gravimetric analysis (TGA) was carried out under nitrogen using a NETZSCH 209 thermal gravimetric analyzer (NETZSCH, Germany). Temperature was raised from room temperature to $700^{\circ} \mathrm{C}$ at a heating rate of $20^{\circ} \mathrm{C} / \mathrm{min}$. $\mathrm{X}$ ray diffraction analysis was conducted using a D8 Advance Powder Diffractometer (Bruker, Germany), experimental conditions: copper target, LynxExe array detector, tube voltage $=40 \mathrm{~kW}$, tube current $=40 \mathrm{~mA}$, and scanning range 2$70^{\circ}$. The compressive strength of RPUF was tested according to $\mathrm{GB} / \mathrm{T} 8813-2008$, with a strain rate of $5 \mathrm{~mm} / \mathrm{min}$; five samples were tested in each group. The limiting oxygen index (LOI) of RPUF was measured in accordance with the standards listed in GB/T2406-93, by using a HC-2 oxygen index tester (Jiangning, China), with five samples in each group. Flame retardancy and smoke suppression were tested on the basis of ASTM D1354-93 and ISQ 5660 by using a standard cone calorimeter (Fire Testing Technology Ltd., $\mathrm{UK})$. The heat flux in the cone calorimeter test was $35 \mathrm{~kW} / \mathrm{m}^{2}$, and two samples were tested in each group. The morphology of composite materials and their residual materials after high-temperature burning was observed using a 1530VP environmental scanning electron microscope (SEM) (LEO Electron Microscopy Ltd., Germany) (Table 2).

\section{Results and Discussion}

3.1. Structural Analysis of DPTP. The IR spectra of KH-550 and DPTP are shown in Figure 1. As can be seen in the figure, the doublet in the KH-550 spectrum at $3200 \mathrm{~cm}^{-1}$, which corresponds to the stretching vibration peak of the primary amine $\left(-\mathrm{NH}_{2}\right)$ group at the end of the molecule, is changed from a doublet to a singlet on the DPTP spectrum. This is due to the fact that the primary amine of $\mathrm{KH}$ 550 is converted to a secondary amine in the reaction. In the DPTP infrared spectrum, peaks at $3053 \mathrm{~cm}^{-1}$ and $690 \mathrm{~cm}^{-1}$ are the absorption peaks of $\mathrm{C}-\mathrm{H}$ in the benzene ring. The peak near $745 \mathrm{~cm}^{-1}$ is the characteristic peak for $\mathrm{P}-\mathrm{N}$, and the absorption peak at $1485 \mathrm{~cm}^{-1}$ corresponds to phenylphosphine. The disappearance of the $\mathrm{P}-\mathrm{Cl}$ absorption peak at $550 \mathrm{~cm}^{-1}$ peak suggests that the $\mathrm{P}-\mathrm{N}$ bond was generated via a chemical reaction between the primary amine 


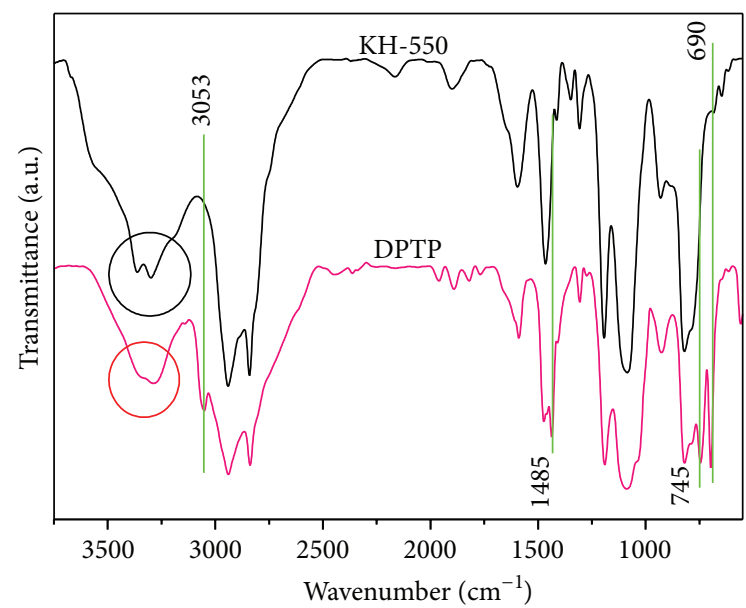

FIGURE 1: IR spectra of KH-550 and DPTP.

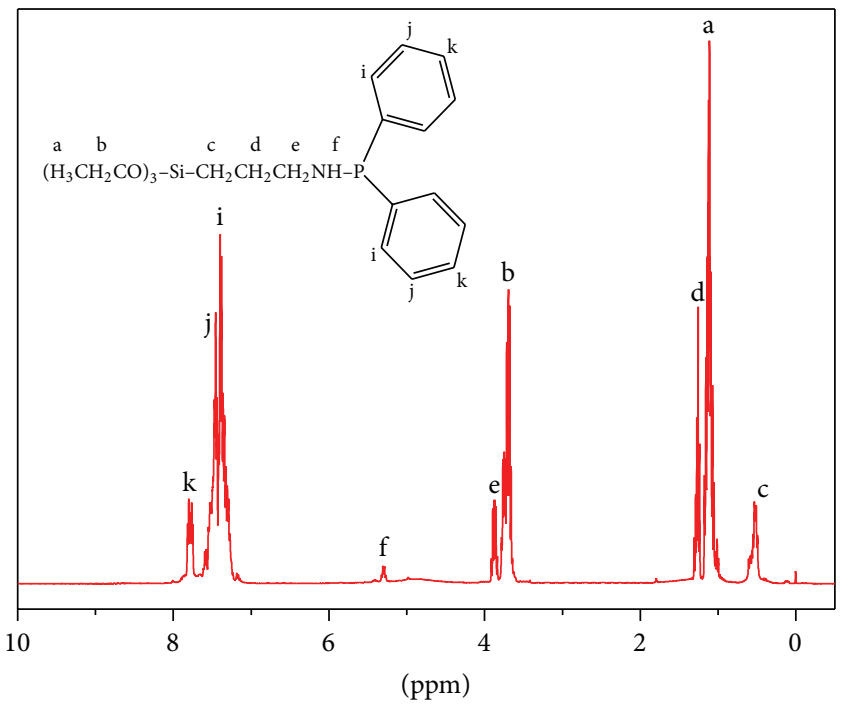

FIgURE 2: ${ }^{1} \mathrm{H}-\mathrm{NMR}$ spectrum of DPTP.

$\left(-\mathrm{NH}_{2}\right)$ and $\mathrm{P}-\mathrm{Cl}$; the aromatic ring out of plane bending is at $745 \mathrm{~cm}^{-1}$. In the ${ }^{1} \mathrm{H}$ NMR of DPTP, the representing phenyl (7.38-7.79 ppm) and $-\mathrm{CH}_{2}-(0.53 \mathrm{ppm}, 1.26 \mathrm{ppm})$ groups can be found in the ${ }^{1} \mathrm{H}$ NMR spectrum of DTES.

3.2. Influence of DPTP on the Mechanical Properties of $R P U F$. Figure 3 shows the compressive strength of RPUF. As shown in the figure, after DPTP was added to RPUF, the compressive strength of the composite material increased initially and then decreased. This is because DPTP contains a silane group and can partly act as a foam stabilizer. The addition of a small amount of DPTP can reduce the surface tension of each component in RPUF, increase the mutual compatibility of each component, and promote the formation and stability of bubbles and can prevent bubble collapse, thus increasing the compressive strength of RPUF. Although DPTP, as a small-molecule flame retardant, can react with RPUF, DPTP can only be connected to the

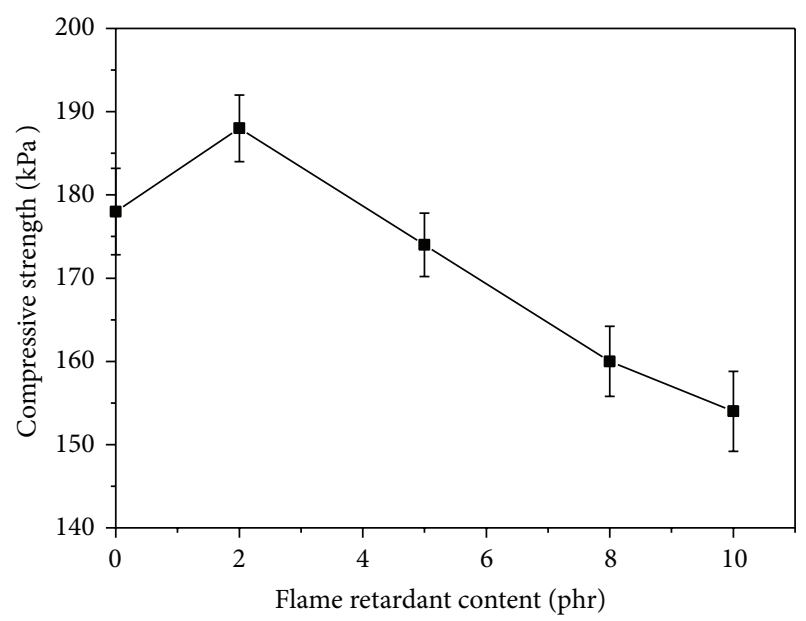

FiguRE 3: Compressive strength of RPUF.

polyurethane macromolecular chain as a small molecule. This damages chain regularity, thus affecting the formation of RPUF into a network structure. Furthermore, the silane group may also have a plasticizing effect [13]. Therefore, when a large amount of DPTP is added, the induced "plasticizing effect" on RPUF causes the bubbles in the foam body to be brittle and is more likely to lead to bubble collapse and deformation.

3.3. Impact of DPTP on the Thermal Stability of RPUF. The TGA curve of RPUF is shown in Figure 4. As shown in the figure, the initial decomposition temperature of pure RPUF was $252^{\circ} \mathrm{C}$, and the char yield at $700^{\circ} \mathrm{C}$ was $17.5 \%$. With increasing phr of DPTP, the char yield of flame-retardant RPUF gradually increased. When $10 \mathrm{phr}$ of DPTP was added, the char yield of flame-retardant RPUF reached 23\%. This demonstrates that the addition of DPTP can form a dense char layer to block heat transfer and the penetration of combustible gases, thus improving the thermal stability and flame retardancy of RPUF composites.

3.4. Impact of DPTP on the Combustion Performance of RPUF. The LOI values of RPUF are shown in Figure 5. The LOI value of pure RPUF was 18.2. After 2, 5, 8, and 10 phr of DPTP were added, the corresponding LOI values were 22.5, 24.6, 26.4, and 27.3, respectively. This suggests that the introduction of DPTP can significantly increase the LOI value of RPUF. As increasing amounts of DPTP were added, the LOI value of the foam gradually increased. This is because the excellent charforming property of DPTP has a synergistic char-forming effect on RPUF, thus significantly increasing the LOI value of composite materials.

Figure 6 depicts the heat release rate, total heat release, smoke release rate, and total smoke release curves of DPTP flame-retardant RPUF composites. The related data are listed in Table 1. As shown in Figure 6, when 2, 5, 8, and $10 \mathrm{phr}$ of DPTP were added to RPUF, the corresponding peak heat release rates (PHRRs) were 273.59, 245.90, 245.51, and $218.92 \mathrm{~kW} / \mathrm{m}^{2}$, respectively. Compared to $361.07 \mathrm{~kW} / \mathrm{m}^{2}$ of 


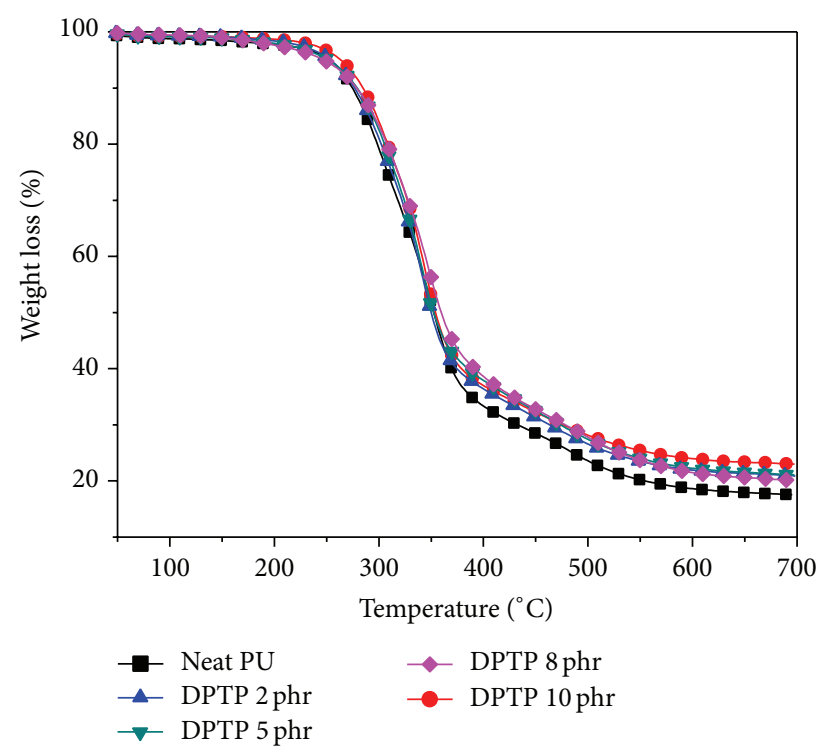

FIGURE 4: TGA curve of RPUF.

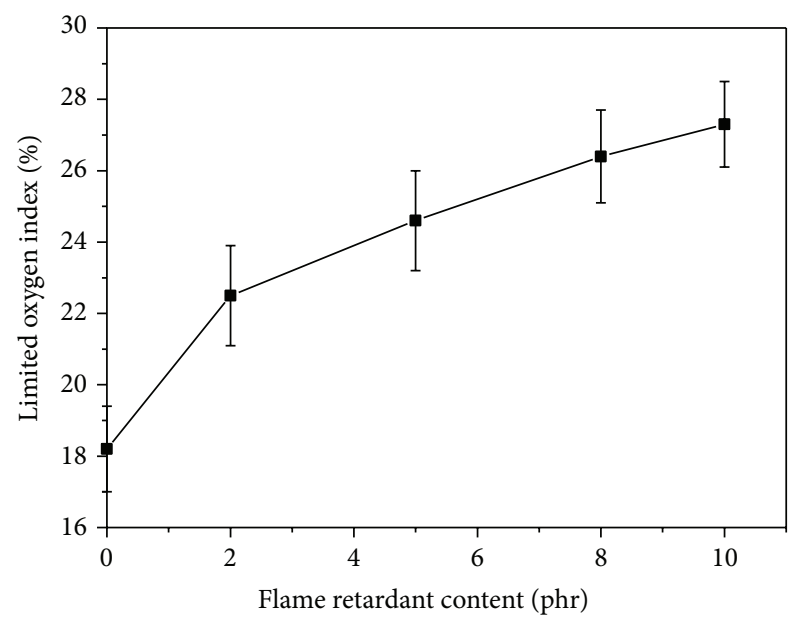

FIGURE 5: LOI values of DPTP flame-retardant RPUF composites.

pure RPUF, the PHRRs were decreased by $24.2 \%, 31.9 \%$, $32.0 \%$, and $39.4 \%$, respectively. The total heat release in the combustion of pure RPUF in $250 \mathrm{~s}$ was $31.6 \mathrm{MJ} / \mathrm{m}^{-2}$. After $2,5,8$, and $10 \mathrm{phr}$ of DPTP were added, the corresponding values of the total heat release during the combustion of RPUF in $250 \mathrm{~s}$ were $22.5,24.3,21.0$, and $23.4 \mathrm{MJ} / \mathrm{m}^{-2}$, respectively, showing a comparative decrease of $28.8 \%, 23.1 \%$, $33.5 \%$, and $25.9 \%$, respectively. This suggests that the addition of DPTP can significantly reduce the exothermic effect and mass loss rate of RPUF during combustion. However, the introduction of DPTP shortens the ignition time of the composite material, since the thermal decomposition temperature of DPTP is lower than that of RPUF macromolecular chains and DPTP has a lower thermal stability. One apparent feature in the combustion of RPUF is that a large amount of thick black smoke is generated. The smoke contains both thermal decomposition products and combustion products.
Under high temperature, polyurethane foam can produce char particles formed from dehydrogenation and particles of compounds containing a small number of hydrogen atoms. Both particles can mix with the small char residue in combustion products. Moreover, alcohol and aldehyde oligomer generated from pyrolysis or agglomerated droplets of small molecules are also mixed in the smoke. Therefore, the combustion of polyurethane foam produces a highly dense smoke with a complicated composition. One important way to reduce the smoke density during the combustion of polyurethane is to increase the amount of condensed products in the combustion of polyurethane foam, that is, to maximize the rate of char residue during combustion and the cohesion of char residue. From the smoke release rate curve, the peak smoke production rate (PSPR) of RPUF was $0.4509 \mathrm{~m}^{2} / \mathrm{s}$. After 2, 5, 8, and $10 \mathrm{phr}$ of DPTP were added, the corresponding PSPRs of composites were reduced to 0.1801 , $0.1952,0.2157$, and $0.2255 \mathrm{~m}^{2} / \mathrm{s}$, respectively. As can be seen from the total smoke release curve, the addition of DPTP can effectively reduce the PSPR and total smoke release of RPUF. After the introduction of DPTP in different ratios, the total smoke release amounts of composite materials combusted within $250 \mathrm{~s}$ were all decreased in comparison to that of pure RPUF. After 2, 5, 8, and $10 \mathrm{phr}$ of DPTP were added, the corresponding amounts of the total smoke release of RPUF were decreased by $52.4 \%, 52.2 \%, 36.3 \%$, and $45.5 \%$, respectively. This is because the molten materials produced during the formation of a dense char layer in DPTP decomposition can cover or wrap the unburned substrate part of RPUF. The evaporation of small residual char particles formed during the combustion of the inner layer of foam was reduced. This can increase the amount of the condensed char layer formed and can reduce the total amount of smoke produced by combustion. The strong and compact char layer produced by DPTP can effectively block smoke penetration and extend the smoke propagation path so that it can reduce PSPR. However, as increasing amount of DPTP was added, PSPR and total smoke release of flame-retardant RPUF increased gradually. This may be due to the fact that the thermal decomposition process produced small molecules, which formed small solid particles. Consequently, the smoke density during the combustion of composite materials increased.

3.5. Flame Retardancy Mechanism. Figure 7 shows the macromorphology of char residues as determined from the cone calorimeter testing of pure cone RPUF and DPTP flame-retardant RPUF composites. Pure RPUF itself has certain char-forming properties and yields a small amount of char residue after combustion. However, this type of char layer is thin and breaks easily. After 2 phr of DPTP was added, the amount of char residue of the RPUF composite increased significantly. After combustion, a continuous char layer could be formed, while the outer layer of char residue was thin, with many cracks and curls on the surface. When increasing amounts of DPTP were added, the char layer became thicker, the amount of char residue increased, the expansion of the char layer was more apparent, the area of the entire char 


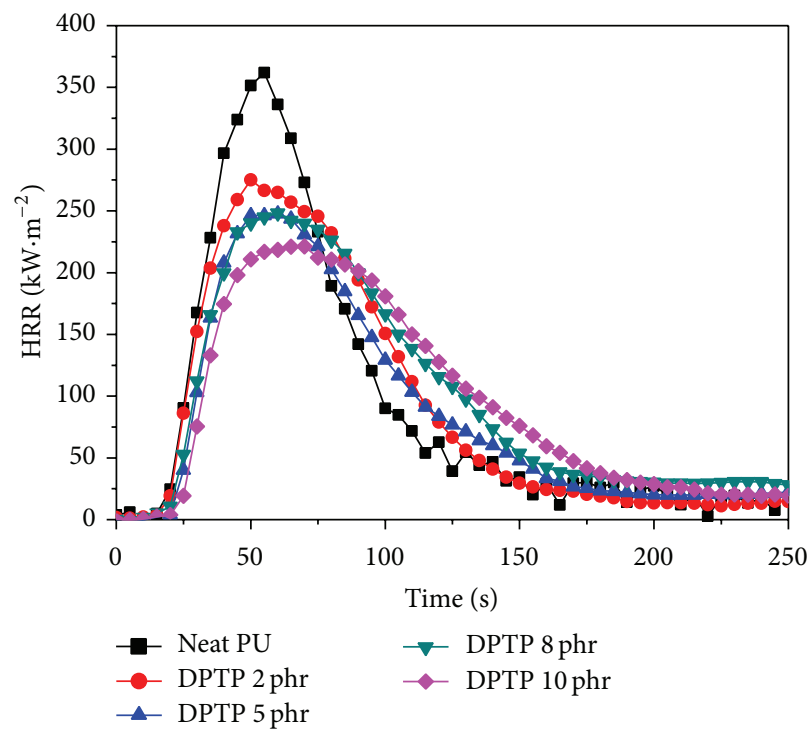

(a)

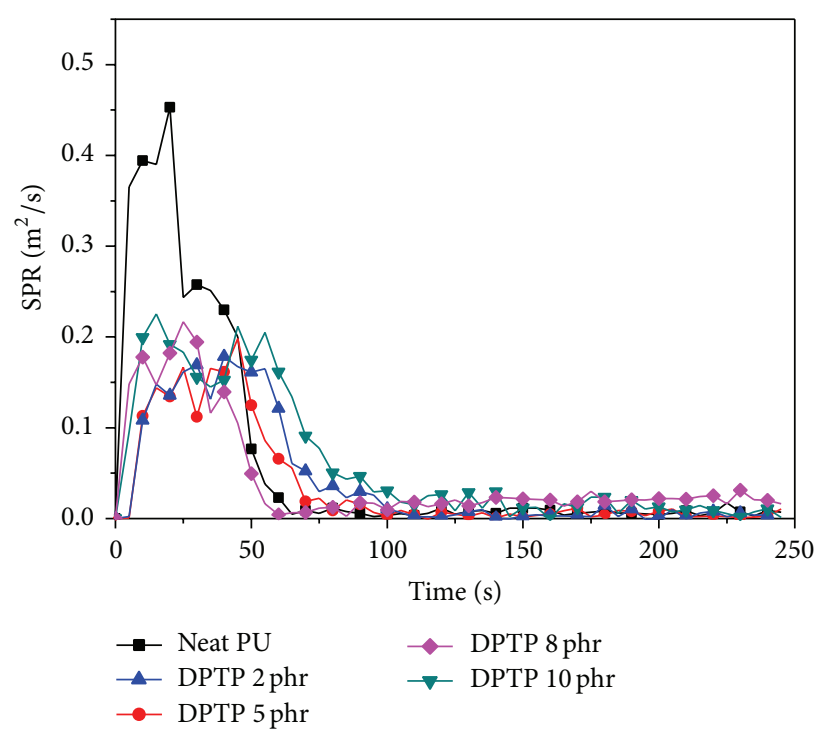

(c)

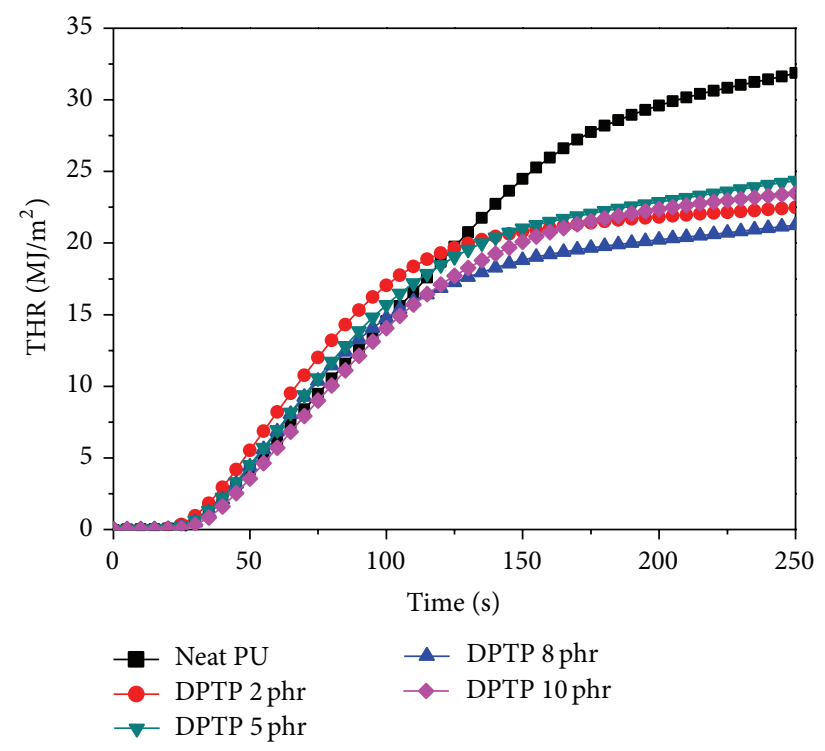

(b)

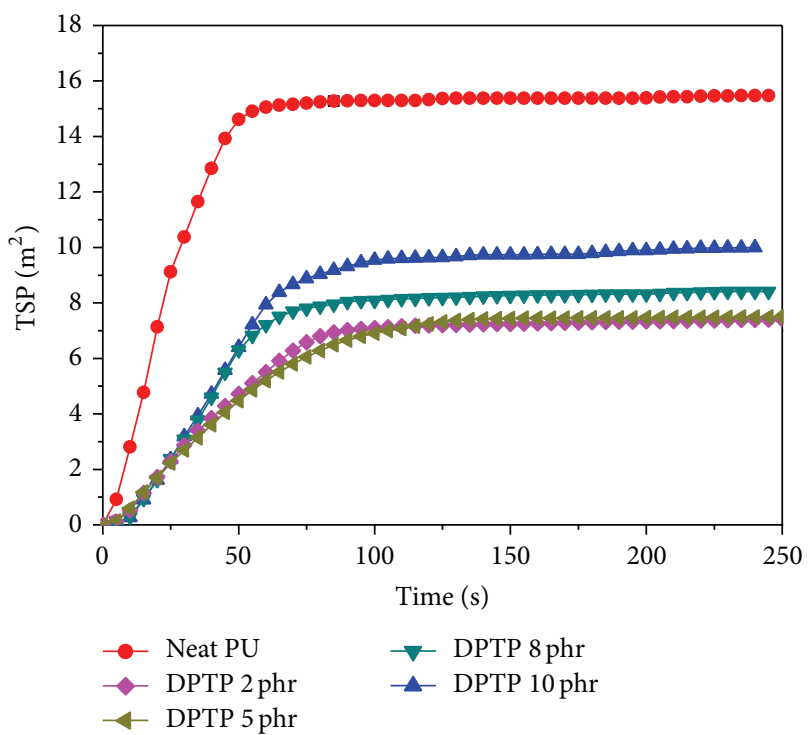

(d)

FIGURE 6: (a) Heat release rate of RPUF, (b) total heat release of RPUF, (c) smoke release rate of RPUF, and (d) total smoke release of RPUF.

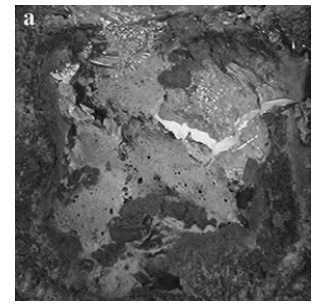

(a)

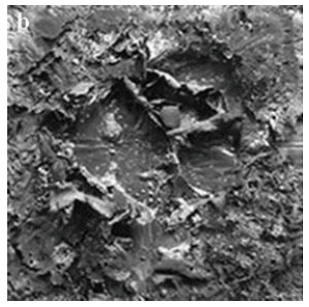

(b)

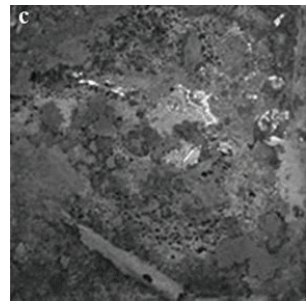

(c)

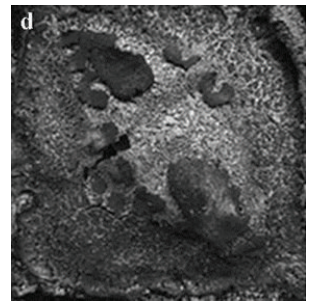

(d)

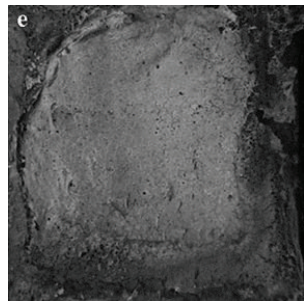

(e)

FIGURE 7: Digital images of char residue after cone calorimeter test: (a) neat PU, (b) DPTP (2 phr), (d) DPTP (5 phr), (e) DPTP (8 phr), and (f) DPTP (10 phr). 


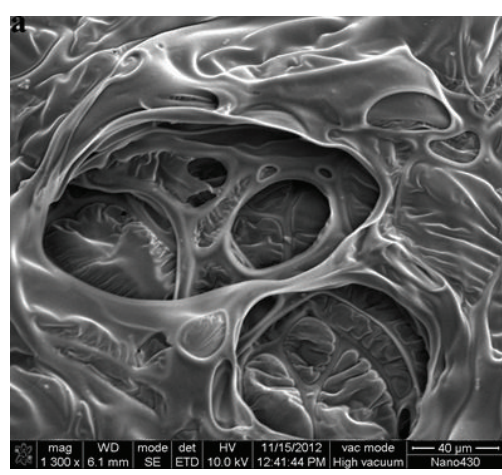

(a)

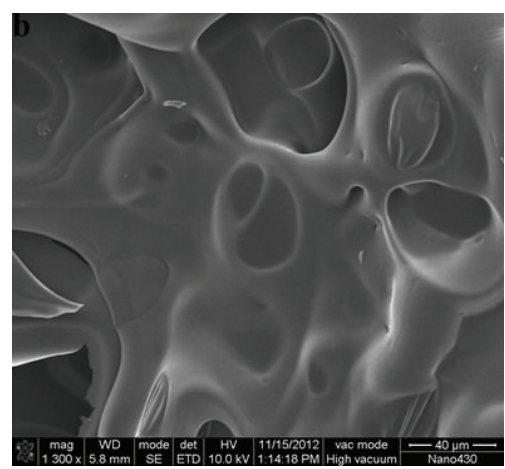

(b)

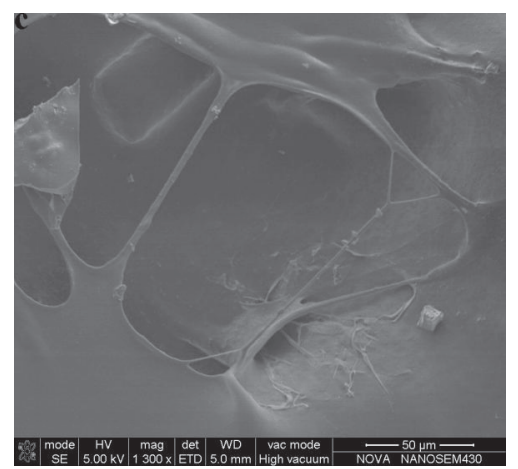

(c)

FIGURE 8: SEM images of char residue after cone calorimeter test: (a) neat PU, (b) DPTP (2 phr), and (c) DPTP (10 phr).

layer gradually increased, and the distribution of the char layer became increasingly uniform. As shown in Figure 7, the char residue formed after the combustion of RPUF with the addition of $10 \mathrm{phr}$ of DPTP formed a complete expanded char layer. Its apparent strength was significantly higher than those of other samples, and no split char layers appeared on the surface $[14,15]$. When compared to a complete layer, cracks were observed between the split layers. The occurrence of cracks will facilitate heat and gas propagation. Therefore, the more the number of cracks on the surface of the char layer is, the worse its barrier effect will be. This complete, compact, and strong char layer can effectively block the propagation of heat and gas, thereby protecting the underlying polymer substrate material.

Figure 8 shows the micromorphology of the inner surface of the char residue obtained after the combustion of pure RPUF and DPTP flame-retardant RPUF composites. As can be seen from the figure, the inner char layer of pure RPUF (Figure 8(a)) has a honeycomb structure, with different sizes of holes. This was caused by a large amount of volatile gas produced from thermal decomposition during the combustion of substrate materials, which broke through the fragile char layer. Figures 8(b) and 8(c) depict the morphology of the char layers of flame-retardant RPUF containing 2 and 10 phr of DPTP. As can be seen from Figure 8(b), many vesicles appeared in the inner layer of the char residue, while there were no holes in these vesicles. This was caused by the foaming effect in the process of char formation from the gases, which were produced during the combustion of the composite materials. Figure 8(c) indicates no apparent vesicles and holes in the inner char layer, suggesting that the char layer in the molten state during char formation had high strength, good thermal insulation, and good gas barrier properties [16-18]. Furthermore, the char layer produced from DPTP added to RPUF was not separated from the char layer produced from the substrate material, yielding a good synergistic effect. This synergistic effect can enhance the strength of the char layer during combustion, reduce the generation of cracks in the char layer, and effectively block heat and mass transfer and the outflow of smoke particles, thus playing an essential role in improving flame retardancy and smoke suppression.

\section{Conclusions}

DPTP added to RPUF can act as a foam stabilizer to enhance the flame retardancy of RPUF. DPTP can significantly increase the char yield of RPUF and can promote char formation during the combustion of RPUF. For RPUF, the addition of DPTP significantly reduced the heat release rate, total heat release, smoke release rate, total smoke release, and average mass loss rate. Moreover, the addition of DPTP significantly increased the LOI value of RPUF. During the combustion of DPTP flame-retardant RPUF composite materials, a complete and thick char layer was formed on the surface and provided insulation. As a result, the RPUF composite material effectively blocked heat and mass transfer and exhibited excellent flame retardancy. It is concluded that the significant improvement in the flame retardancy of RPUF may be attributed to the interaction between aminopropyl triethoxysilane and chlorodiphenyl phosphine, which increases both the quantity and thermal stability of the char. The enhanced char layer serves as a perfect shell to accumulate the pyrolytic gases during combustion of RPUF. Thus, the flame retardant was enhanced.

\section{Conflict of Interests}

The authors declare that there is no conflict of interests regarding the publication of this paper.

\section{Acknowledgments}

The authors thank the Fundamental Research for the Major Projects in Guangdong Province by Ministry of Education Research (2011A090200051), the Guangdong-Hong kong Breakthrough Bidding Project in Key Areas (2012BZ100054), and the Joint Funds of the National Natural of China (U1134005). 


\section{References}

[1] N. Athanasopoulos, A. Baltopoulos, M. Matzakou, A. Vavouliotis, and V. Kostopoulos, "Electrical conductivity of polyurethane/MWCNT nanocomposite foams," Polymer Composites, vol. 33, no. 8, pp. 1302-1312, 2012.

[2] Y. Li and A. J. Ragauskas, "Ethanol organosolv ligninbased rigid polyurethane foam reinforced with cellulose nanowhiskers," RSC Advances, vol. 2, no. 8, pp. 3347-3351, 2012.

[3] S. Tan, T. Abraham, D. Ference, and C. W. MacOsko, "Rigid polyurethane foams from a soybean oil-based Polyol," Polymer, vol. 52, no. 13, pp. 2840-2846, 2011.

[4] A. R. Tarakcilar, "The effects of intumescent flame retardant including ammonium polyphosphate/pentaerythritol and fly ash fillers on the physicomechanical properties of rigid polyurethane foams," Journal of Applied Polymer Science, vol. 120, no. 4, pp. 2095-2102, 2011.

[5] X. G. Zhang, L. L. Ge, W. Q. Zhang, J. H. Tang, L. Ye, and Z. M. Li, "Expandable graphite-methyl methacrylate-acrylic acid copolymer composite particles as a flame retardant of rigid polyurethane foam," Journal of Applied Polymer Science, vol. 122, no. 2, pp. 932-941, 2011.

[6] Z. Zheng, J. Yan, H. Sun et al., "Preparation and characterization of microencapsulated ammonium polyphosphate and its synergistic flame-retarded polyurethane rigid foams with expandable graphite," Polymer International, vol. 63, no. 1, pp. 84-92, 2014.

[7] H.-J. Duan, H.-Q. Kang, W.-Q. Zhang, X. Ji, Z.-M. Li, and J.$\mathrm{H}$. Tang, "Core-shell structure design of pulverized expandable graphite particles and their application in flame-retardant rigid polyurethane foams," Polymer International, vol. 63, no. 1, pp. 72-83, 2014.

[8] F. Feng and L. Qian, "The flame retardant behaviors and synergistic effect of expandable graphite and dimethyl methylphosphonate in rigid polyurethane foams," Polymer Composites, vol. 35, no. 2, pp. 301-309, 2014.

[9] X.-Y. Meng, L. Ye, X.-G. Zhang et al., "Effects of expandable graphite and ammonium polyphosphate on the flame-retardant and mechanical properties of rigid polyurethane foams," Journal of Applied Polymer Science, vol. 114, no. 2, pp. 853-863, 2009.

[10] N. Usta, "Investigation of fire behavior of rigid polyurethane foams containing fly ash and intumescent flame retardant by using a cone calorimeter," Journal of Applied Polymer Science, vol. 124, no. 4, pp. 3372-3382, 2012.

[11] V. B. Veronese, R. K. Menger, M. M. D. C. Forte, and C. L. Petzhold, "Rigid polyurethane foam based on modified vegetable oil," Journal of Applied Polymer Science, vol. 120, no. 1, pp. 530-537, 2011.

[12] L. Zhang, M. Zhang, Y. Zhou, and L. Hu, "The study of mechanical behavior and flame retardancy of castor oil phosphate-based rigid polyurethane foam composites containing expanded graphite and triethyl phosphate," Polymer Degradation and Stability, vol. 98, no. 12, pp. 2784-2794, 2013.

[13] Y. H. Kim, M. J. Kang, G. P. Park, S. D. Park, S. B. Kim, and W. N. Kim, "Effects of liquid-type silane additives and organoclay on the morphology and thermal conductivity of rigid polyisocyanurate-polyurethane foams," Journal of Applied Polymer Science, vol. 124, no. 4, pp. 3117-3123, 2012.

[14] W. Zhao, B. Li, M. Xu, F. Liu, and L. Guan, "Synthesis of a phenylene phenyl phosphine oligomer and its flame retardancy for polycarbonate," Journal of Applied Polymer Science, vol. 127, no. 4, pp. 2855-2866, 2013.
[15] H. Ren, J. Sun, B. Wu, and Q. Zhou, "Synthesis and properties of a phosphorus-containing flame retardant epoxy resin based on bis-phenoxy (3-hydroxy) phenyl phosphine oxide," Polymer Degradation and Stability, vol. 92, no. 6, pp. 956-961, 2007.

[16] X. H. Zhang, F. Liu, S. Chen, and G. R. Qi, "Novel flame retardant thermosets from nitrogen-containing and phosphoruscontaining epoxy resins cured with dicyandiamide," Journal of Applied Polymer Science, vol. 106, no. 4, pp. 2391-2397, 2007.

[17] G. Durga and A. K. Narula, "Synthesis and characterization of diamide-diimide-diamines based on p-amino benzoic acid and their curing and thermal behavior with epoxy blends containing phosphorus/silicon in the main chain," Journal of Applied Polymer Science, vol. 124, no. 5, pp. 3685-3694, 2012.

[18] O. Fischer, D. Pospiech, A. Korwitz et al., "Synthesis and properties of phosphorus polyesters with systematically altered phosphorus environment," Polymer Degradation and Stability, vol. 96, no. 12, pp. 2198-2208, 2011. 

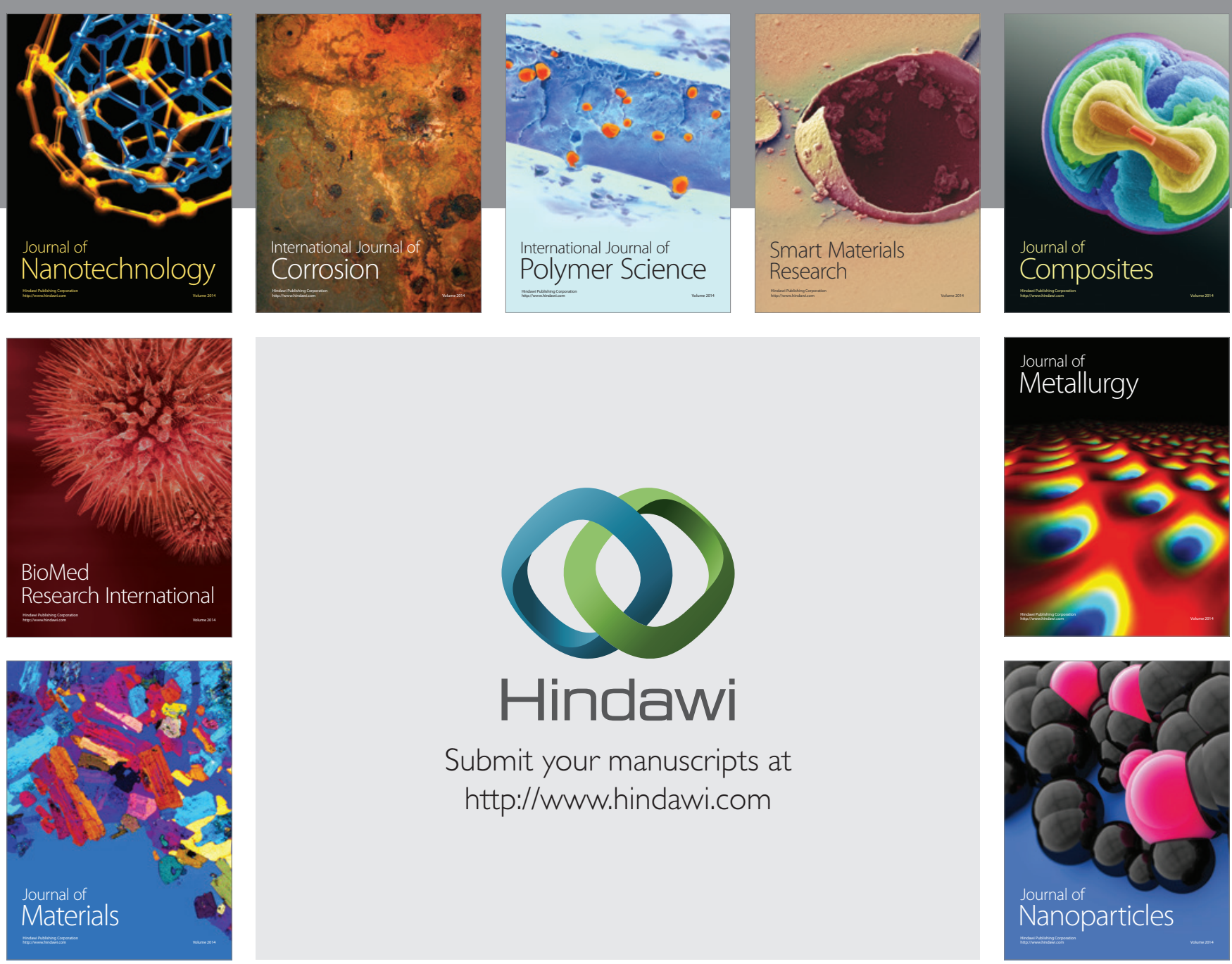

Submit your manuscripts at http://www.hindawi.com
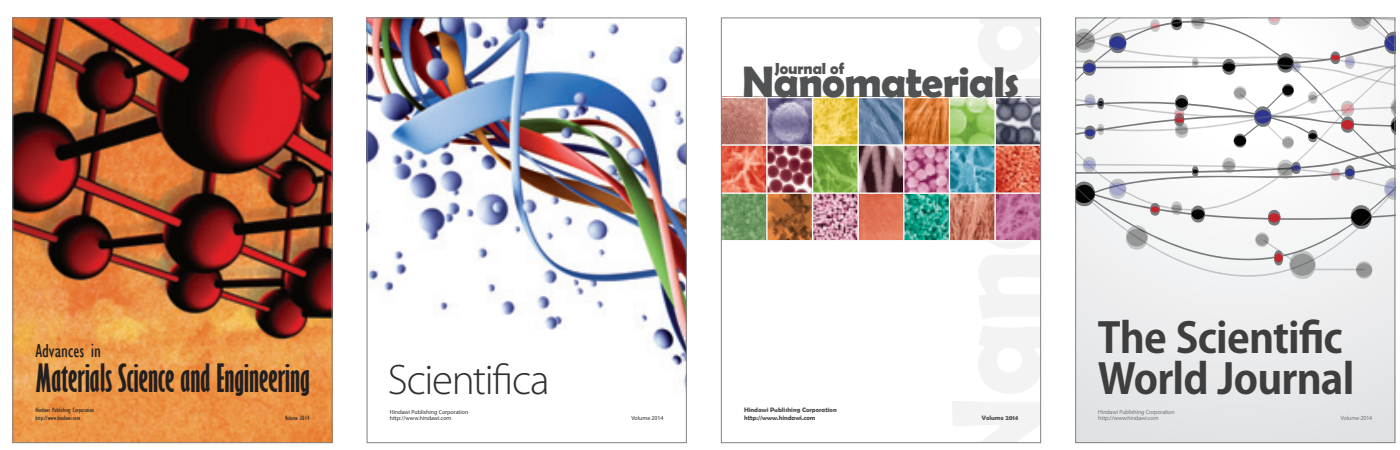

\section{The Scientific World Journal}
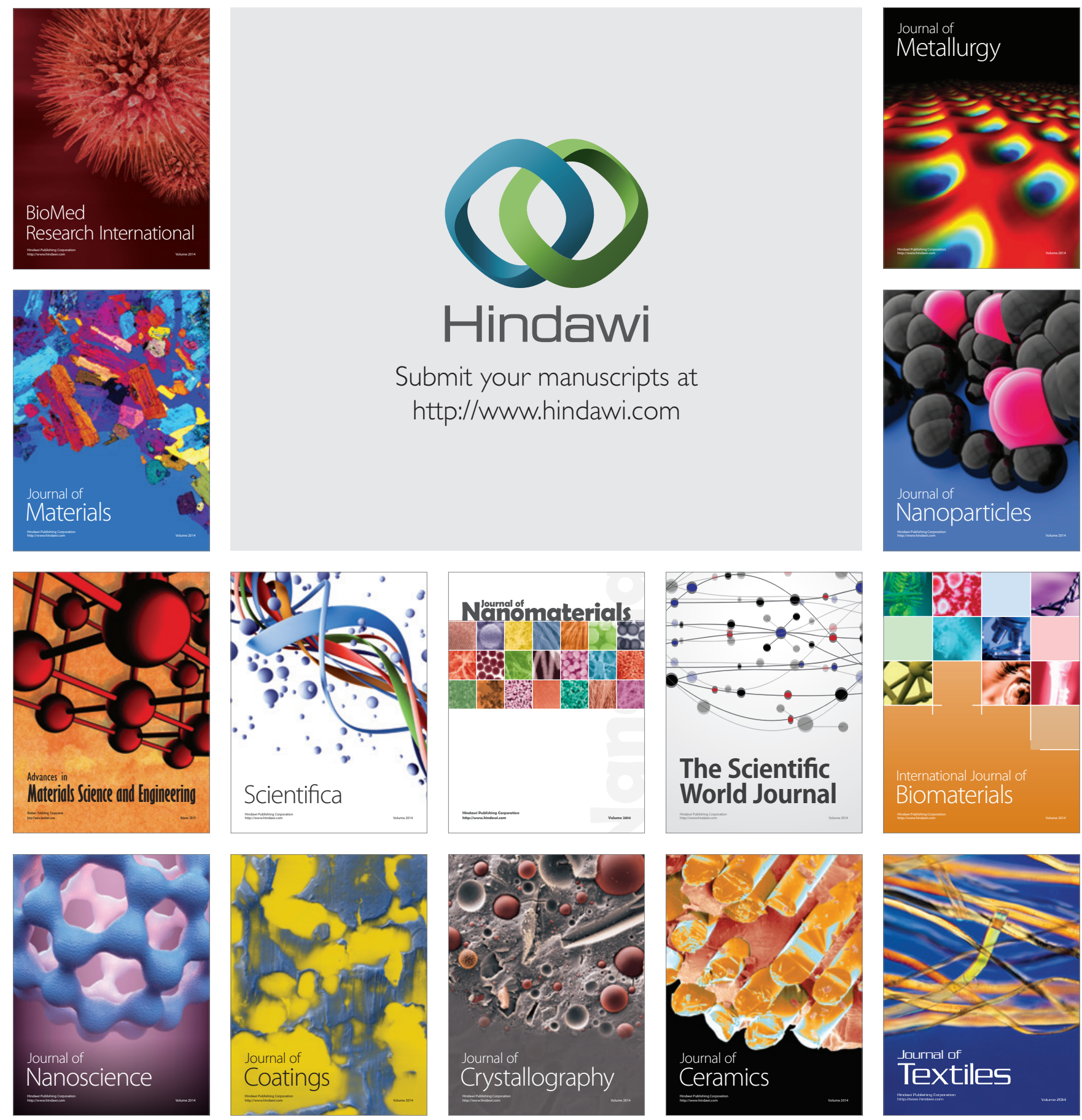